Bull. Austral. Math. Soc.

VoL. 75 (2007) [221-227]

\title{
ON THE DISTRIBUTION OF ANGLES OF THE SALIÉ SUMS
}

IGOR E. SHPARLINSKI

For a prime $p$ and integers $a$ and $b$, we consider Salié sums

$$
S_{p}(a, b)=\sum_{x=1}^{p-1} \chi_{2}(x) \exp (2 \pi i(a x+b \bar{x}) / p),
$$

where $\chi_{2}(x)$ is a quadratic character and $\bar{x}$ is the modular inversion of $x$, that is, $x \bar{x}$ $\equiv 1(\bmod p)$. One can naturally associate with $S_{p}(a, b)$ a certain angle $\vartheta_{p}(a, b)$ $\epsilon[0, \pi]$. We show that, for any fixed $\varepsilon>0$, these angles are uniformly distributed in $[0, \pi]$ when $a$ and $b$ run over arbitrary sets $\mathcal{A}, \mathcal{B} \subseteq\{0,1, \ldots, p-1\}$ such that there are at least $p^{1+\varepsilon}$ quadratic residues modulo $p$ among the products $a b$, where $(a, b) \in \mathcal{A} \times \mathcal{B}$.

\section{INTRODUCTION}

For a prime $p \geqslant 3$ and integers $a$ and $b$, we consider Salié sums

$$
S_{p}(a, b)=\sum_{x=1}^{p-1} \chi_{2}(x) \mathbf{e}_{p}(a x+b \bar{x}),
$$

where $\chi_{2}(x)$ is a quadratic character, $\bar{x}$ is the modular inversion of $x$, that is, $x \bar{x} \equiv 1$ $(\bmod p)$, and

$$
\mathbf{e}_{p}(z)=\exp (2 \pi i z / p)
$$

One can naturally associate with $S_{p}(a, b)$ a certain angle $\vartheta_{p}(a, b)$. It is known, see $[7,15]$ that for integers $a$ and $b$ with $\operatorname{gcd}(a b, p)=1$ we have

$$
S_{p}(a, b)=G_{p}(a) \sum_{\substack{u=1 \\ u^{2} \equiv 4 a b(\bmod p)}}^{p-1} e_{p}(u)
$$

where

$$
G_{p}(a)=\sum_{x=0}^{p-1} \mathrm{e}_{p}\left(a x^{2}\right)
$$

Received 4th September, 2006

Copyright Clearance Centre, Inc. Serial-fee code: 0004-9727/07 \$A2.00+0.00. 
is the Gauss sum. Thus $S_{p}(a, b)$ vanishes if $\chi_{2}(a b)=-1$ and

$$
S_{p}(a, b)=G_{p}(a) \cos \left(\frac{2 \pi u_{p}(a, b)}{p}\right)=G_{p}(b) \cos \left(\frac{2 \pi u_{p}(a, b)}{p}\right)
$$

if $\chi_{2}(a b)=1$, where $u_{p}(a, b)$ is the smallest solution to the following congruence:

$$
u^{2} \equiv 4 a b \quad(\bmod p), \quad 1 \leqslant u \leqslant p-1 .
$$

Thus it is natural to say that

$$
\vartheta_{p}(a, b)=\frac{2 \pi u_{p}(a, b)}{p}
$$

is the angle of the Salié sum $S_{p}(a, b)$.

Duke, Friedlander and Iwaniec [4] and Tóth [17] using very deep arguments, show that if $a$ and $b$ are fixed integers, then the sequence of the angles $\vartheta_{p}(a, b)$ is uniformly distributed in the interval $[0, \pi]$ when $p$ runs through the primes such that $a b$ is a quadratic residue modulo $p$.

Here we show that a similar result also holds for the case when a sufficiently large prime $p$ is fixed and $a$ and $b$ run through arbitrary sets of integers $\mathcal{A}$ and $\mathcal{B}$ which both have some sufficiently many quadratic residues or non-residues. For example, $\mathcal{A}$ and $\mathcal{B}$, could consist of consecutive integers each (for arbitrary $\varepsilon>0$ ).

It is useful to recall, that Kloosterman sums

$$
K_{p}(a, b)=\sum_{x=1}^{p-1} \mathbf{e}_{p}(a x+b \bar{x}),
$$

which are very close relatives of Salié sums, exhibit a very different behaviour described by the Sato-Tate conjecture. See $[1,3,5,6,8,9,10,12,13,14,16]$ for various modifications and generalisations of this conjecture and further references.

Throughout the paper, the implied constants in the symbols ' $O$ ', and ' $\ll$ ' are absolute. We recall that the notations $U=O(V)$ and $U \ll V$ are both equivalent to the assertion that the inequality $|U| \leqslant c V$ holds for some constant $c>0$.

\section{Distribution of Square Roots of Products}

It is clear that the question of studying $\vartheta_{p}(a, b)$ with $a \in \mathcal{A}$ and $b \in \mathcal{B}$ is equivalent to the question of studying the distribution of solutions to the congruence (1).

Given two sets $\mathcal{A}, \mathcal{B} \subseteq\{0,1, \ldots, p-1\}$ we study the uniformity of distribution of the sequence of fractions $u / p$, where $u$ runs through all solutions to the congruence (1), taken over all pairs $(a, b) \in \mathcal{A} \times \mathcal{B}$. That is, for a real $\gamma \in[0,1]$ we consider the counting function

$$
N_{p, \gamma}(\mathcal{A}, \mathcal{B})=\sum_{a \in \mathcal{A}} \sum_{b \in \mathcal{B}} \sum_{\substack{u=1 \\ u^{2} \equiv 4 a b(\bmod p) \\ u / p \leqslant \gamma}}^{p-1} 1
$$


and put for brevity

$$
N_{p}(\mathcal{A}, \mathcal{B})=N_{p, 1}(\mathcal{A}, \mathcal{B})
$$

One sees that $N_{p}(\mathcal{A}, \mathcal{B})$ is twice the number of pairs $(a, b) \in \mathcal{A} \times \mathcal{B}$ with $\chi_{2}(a b)=1$.

We now define the discrepancy of the sequence of solutions to the congruence (1) for $(a, b) \in \mathcal{A} \times \mathcal{B}$ :

$$
D_{p}(\mathcal{A}, \mathcal{B})=\max _{0 \leqslant \gamma<1}\left|\frac{N_{p, \gamma}(\mathcal{A}, \mathcal{B})}{N_{p}(\mathcal{A}, \mathcal{B})}-\gamma\right| .
$$

Theorem 1. For any two sets $\mathcal{A}, \mathcal{B} \subseteq\{0,1, \ldots, p-1\}$, we have

$$
D_{p}(\mathcal{A}, \mathcal{B}) \ll \sqrt{\frac{p}{N_{p}(\mathcal{A}, \mathcal{B})}} \log p .
$$

Proof: We fix some $\gamma \in[0,1)$ and note that for $h=\lfloor\gamma p\rfloor$, we can write $N_{p, \gamma}(\mathcal{A}, \mathcal{B})$ as

$$
N_{p, \gamma}(\mathcal{A}, \mathcal{B})=\sum_{a \in \mathcal{A}} \sum_{b \in \mathcal{B}} \sum_{\substack{u=1 \\ u^{2} \equiv 4 a b}}^{h} 1=\sum_{\nu=0}^{1} \sum_{a \in \mathcal{A}_{\nu}} \sum_{b \in \mathcal{B}_{\nu}} \sum_{\substack{u=1 \\ u^{2} \equiv 4 a b}}^{h} 1
$$

where $\mathcal{A}_{0}, \mathcal{A}_{1}$ and $\mathcal{B}_{0}, \mathcal{B}_{1}$ are subsets of quadratic residues and non-residues among the elements of $\mathcal{A}$ and $\mathcal{B}$, respectively.

Let $\mathcal{X}$ be the set of all $p-1$ multiplicative characters modulo $p$. We recall the identity

$$
\frac{1}{p-1} \sum_{\chi \in \mathcal{X}} \chi(c)= \begin{cases}1 & \text { if } c \equiv 1 \quad(\bmod p) \\ 0 & \text { otherwise }\end{cases}
$$

which holds for any integer $c$. Using (3), we write

$$
\begin{aligned}
\sum_{a \in \mathcal{A}_{\nu}} \sum_{b \in \mathcal{B}_{\nu}} \sum_{\substack{u=1 \\
u^{2} \equiv 4 a b}} 1 & =\sum_{a \in \mathcal{A}_{\nu}} \sum_{b \in \mathcal{B}_{\nu}} \sum_{u=1}^{h} \frac{1}{p-1} \sum_{\chi \in \mathcal{X}} \chi\left(4 a b \bar{u}^{2}\right) \\
& =\frac{1}{p-1} \sum_{\chi \in \mathcal{X}} \sum_{a \in \mathcal{A}_{\nu}} \sum_{b \in B_{\nu}} \sum_{u=1}^{h} \chi\left(4 a b \bar{u}^{2}\right)
\end{aligned}
$$

for $\nu=0,1$. Clearly for for $\chi=\chi_{0}$ (the principal character) and also for $\chi=\chi_{2}$ we have $\chi\left(4 a b \bar{u}^{2}\right)=1$ over the whole area of summation over $a, b$ and $u$. Hence,

$$
\begin{aligned}
& \sum_{a \in \mathcal{A}_{\nu}} \sum_{b \in B_{\nu}} \sum_{\substack{u=1 \\
u^{2} \equiv 4 a b(\bmod p)}}^{h} 1 \\
& \quad=2 \# \mathcal{A}_{\nu} \# \mathcal{B}_{\nu} \frac{h}{p-1}+\frac{1}{p-1} \sum_{\substack{x \in \mathcal{X} \\
x \neq \chi_{0}, \chi_{2}}} \chi(4) \sum_{a \in \mathcal{A}_{\nu}} \chi(a) \sum_{b \in B_{\nu}} \chi(b) \sum_{u=1}^{h} \chi\left(\bar{u}^{2}\right) .
\end{aligned}
$$


If $\chi \neq \chi_{0}, \chi_{2}$ then $\psi(u)=\chi\left(\bar{u}^{2}\right)$ is a nonprincipal multiplicative character and by the Polya-Vinogradov bound, see [7, Theorems 12.5], we obtain

$$
\sum_{u=1}^{h} \chi\left(\bar{u}^{2}\right) \ll p^{1 / 2} \log p .
$$

Therefore,

$$
\sum_{a \in \mathcal{A}_{\nu}} \sum_{b \in \mathcal{B}_{\nu}} \sum_{\substack{u=1 \\ u^{2} \equiv 4 a b}}^{h} 1=2 \# \mathcal{A}_{\nu} \# \mathcal{B}_{\nu} \frac{h}{p-1}+O\left(W_{\nu} p^{-1 / 2} \log p\right)
$$

where

$$
W_{\nu}=\sum_{\chi \in \mathcal{X}}\left|\sum_{a \in \mathcal{A}_{\nu}} \chi(a)\right|\left|\sum_{b \in B_{\nu}} \chi(b)\right|
$$

(Note that we have again extended the summation over all $\chi \in \mathcal{X}$.)

Furthermore, using the Cauchy inequality, we obtain

$$
W_{\nu}^{2} \leqslant \sum_{\chi \in \mathcal{X}}\left|\sum_{a \in \mathcal{A}_{\nu}} \chi(a)\right|^{2} \sum_{\chi \in \mathcal{X}}\left|\sum_{b \in \mathcal{B}_{\nu}} \chi(b)\right|^{2}
$$

We recall that if $\operatorname{gcd}(c, q)=1$, then for the conjugated character $\bar{\chi}$ we have $\bar{\chi}(c)=\chi(\bar{c})$.

Therefore, by (3)

$$
\sum_{\chi \in \mathcal{X}}\left|\sum_{a \in \mathcal{A}_{\nu}} \chi(a)\right|^{2}=\sum_{\chi \in \mathcal{X}} \sum_{a_{1}, a_{2} \in \mathcal{A}_{\nu}} \chi\left(a_{1} a_{2}\right)=\sum_{a_{1}, a_{2} \in \mathcal{A}_{\nu}} \sum_{\chi \in \mathcal{X}} \chi\left(a_{1} \overline{a_{2}}\right)=(p-1) \# \mathcal{A}_{\nu}
$$

and similarly

$$
\sum_{\chi \in \mathcal{X}}\left|\sum_{b \in \mathcal{B}_{\nu}} \chi(b)\right|^{2}=(p-1) \# \mathcal{B}_{\nu}
$$

We now infer from (5) that

$$
W_{\nu} \ll p \sqrt{\# \mathcal{A}_{\nu} \# \mathcal{B}_{\nu}}
$$

which after substitution into (4) leads to the bound

$$
\sum_{a \in \mathcal{A}_{\nu}} \sum_{b \in \mathcal{B}_{\nu}} \sum_{\substack{u=1 \\ u^{2} \equiv 4 a b}}^{h} 1=2 \# \mathcal{A}_{\nu} \# \mathcal{B}_{\nu} \frac{h}{p-1}+O\left(\sqrt{\# \mathcal{A}_{\nu} \# \mathcal{B}_{\nu} p} \log p\right)
$$

for $\nu=0,1$. Furthermore, as we have mentioned,

$$
N_{p}(\mathcal{A}, \mathcal{B})=2\left(\# \mathcal{A}_{0} \# \mathcal{B}_{0}+\# \mathcal{A}_{1} \# \mathcal{B}_{1}\right)
$$


Hence, after substituting (6) in (2) we obtain

$$
N_{p, \gamma}(\mathcal{A}, \mathcal{B})=N_{p}(\mathcal{A}, \mathcal{B}) \frac{h}{p-1}+O\left(\sqrt{N_{p}(\mathcal{A}, \mathcal{B}) p} \log p\right)
$$

Since

$$
\begin{aligned}
N_{p}(\mathcal{A}, \mathcal{B}) \frac{h}{p-1}-\gamma N_{p}(\mathcal{A}, \mathcal{B}) & \ll N_{p}(\mathcal{A}, \mathcal{B})\left(\frac{\gamma p+O(1)}{p-1}-\gamma\right) \\
& \ll N_{p}(\mathcal{A}, \mathcal{B}) p^{-1} \ll \sqrt{N_{p}(\mathcal{A}, \mathcal{B}) p}
\end{aligned}
$$

the desired result follows.

\section{Angles of Salié Sums}

Let for $0 \leqslant \alpha \leqslant \pi$ and two sets $\mathcal{A}, \mathcal{B} \subseteq\{0,1, \ldots, p-1\}$, we denote by $T_{p, \alpha}(\mathcal{A}, \mathcal{B})$ the number of $(a, b) \in \mathcal{A} \times \mathcal{B}$ with $\chi_{2}(a b)=1$ for which

$$
\vartheta_{p}(a, b) \leqslant \alpha,
$$

and put for brevity

$$
T_{p}(\mathcal{A}, \mathcal{B})=T_{p, \pi}(\mathcal{A}, \mathcal{B})
$$

We now define the discrepancy of the sequence of solutions to the congruence (1) for $(a, b) \in \mathcal{A} \times \mathcal{B}$ :

$$
\Delta_{p}(\mathcal{A}, \mathcal{B})=\max _{0 \leqslant \alpha<\pi}\left|\frac{T_{p, \alpha}(\mathcal{A}, \mathcal{B})}{T_{p}(\mathcal{A}, \mathcal{B})}-\alpha\right| .
$$

THEOREM 2. For any two sets $\mathcal{A}, \mathcal{B} \subseteq\{0,1, \ldots, p-1\}$, we have

$$
\Delta_{p}(\mathcal{A}, \mathcal{B}) \ll \sqrt{\frac{p}{T_{p}(\mathcal{A}, \mathcal{B})}} \log p
$$

Proof: Clearly

$$
T_{p, \alpha}(\mathcal{A}, \mathcal{B})=N_{p, \alpha / 2 \pi}(\mathcal{A}, \mathcal{B})
$$

for $0 \leqslant \alpha<\pi$ and also

$$
T_{p, \pi}(\mathcal{A}, \mathcal{B})=N_{p, 1 / 2}(\mathcal{A}, \mathcal{B})=\frac{1}{2} N_{p}(\mathcal{A}, \mathcal{B})
$$

Using Theorem 1 we immediately obtain the desired result. 


\section{Comments}

Clearly the asymptotic formulas of Theorems 1 and 2 are nontrivial under the condition

$$
N_{p}(\mathcal{A}, \mathcal{B}) \geqslant p^{1+\varepsilon}
$$

for any $\varepsilon>0$ and sufficiently large $p$.

For example, if for some fixed $\varepsilon>0$, the sets $\mathcal{A}$ and $\mathcal{B}$ consist of at least $p^{1 / 4+\varepsilon}$ consecutive integers each, then by the Burgess bound, see [7, Theorems 12.6],

$$
N_{p}(\mathcal{A}, \mathcal{B})=\left(\frac{1}{2}+o(1)\right) \# \mathcal{A} \# \mathcal{B} \text {. }
$$

Furthermore, it follows from [2] that if for some fixed $\varepsilon>0$, the sets $\mathcal{A}$ and $\mathcal{B}$ consist of at least $p^{1 / 4 e^{1 / 2}+\varepsilon}$ consecutive integers each, then, for sufficiently large $p$,

$$
N_{p}(\mathcal{A}, \mathcal{B}) \geqslant c(\varepsilon) \# \mathcal{A} \# \mathcal{B}
$$

where $c(\varepsilon)>0$ depends only on $\varepsilon$. Thus, if in addition we also have $\# \mathcal{A} \# \mathcal{B} \geqslant p^{1+\varepsilon}$ then the condition (7) is satisfied.

On the other hand, an example of the sets

$$
\mathcal{A}=\mathcal{B}=\left\{a^{2} \mid 1 \leqslant a \leqslant 0.5 p^{1 / 2}\right\}
$$

for which all solutions to (1) are outside of the interval $[p / 4,3 p / 4]$, shows the limitations of what can be proven.

\section{REFERENCES}

[1] A. Adolphson, 'On the distribution of angles of Kloosterman sums', J. Reine Angew. Math. 395 (1989), 214-220.

[2] W.D. Banks, M.Z. Garaev and I.E. Shparlinski, 'Density of non-residues in short intervals', (preprint 2006).

[3] C.-L. Chai and W.-C.W. Li, 'Character sums, automorphic forms, equidistribution, and Ramanujan graphs. I: The Kloosterman sum conjecture over function fields', Forum Math. 15 (2003), 679-699.

[4] W. Duke, J.B. Friedlander and H. Iwaniec, 'Equidistribution of roots of a quadratic congruence to prime moduli', Ann. of Math. 141 (1995), 423-441.

[5] É. Fouvry and P. Michel, 'Sommes de modules de sommes d'exponentielles', Pacific J. Math. 209 (2003), 261-288.

[6] É. Fouvry and P. Michel, 'Sur le changement de signe des sommes de Kloosterman', Ann. Math. (to appear).

[7] H. Iwaniec and E. Kowalski, Analytic number theory (American Mathematical Society, Providence, RI, 2004). 
[8] N.M. Katz, Gauss sums, Kloosterman sums, and monodromy groups (Princeton Univ. Press, Princeton, NJ, 1988).

[9] N.M. Katz and P. Sarnak, Random matrices, Frobenius eigenvalues, and monodromy (Amer. Math. Soc, Providence, RI, 1999).

[10] G. Laumon, 'Exponential sums and l-adic cohomology: A survey', Israel J. Math. 120 (2000), 225-257.

[11] R. Lidl and H. Niederreiter, Finite fields (Cambridge University Press, Cambridge, 1997).

[12] P. Michel, 'Autour de la conjecture de Sato-Tate pour les sommes de Kloosterman, II', Duke Math. J. 92 (1998), 221-254.

[13] P. Michel, 'Minoration de sommes d'exponentielles', Duke Math. J. 95 (1998), 227-240.

[14] H. Niederreiter, 'The distribution of values of Kloosterman sums', Arch. Math. 56 (1991), 270-277.

[15] P. Sarnak, Some applications of modular forms (Cambridge University Press, Cambridge, 1990).

[16] I.E. Shparlinski, 'On the distribution of Kloosterman sums', Proc. Amer. Math. Soc. (to appear).

[17] Á. Tóth, 'Roots of quadratic congruences', Internat. Math. Res. Notices 2000 (2000), 719-739.

Department of Computing

Macquarie University

Sydney, NSW 2109

Australia

e-mail: igor@ics.mq.edu.au 